\title{
Analysis of the Innovation Strategy of Civilian Battalion Hospital Financial Management under "Internet +" Environment
}

\author{
SUN Limin \\ Department of management, Changsha Medical University \\ sunlimincs@126.com
}

\begin{abstract}
Keywords: "Internet +" environment; Private hospitals; Financial management; Innovation strategy Abstract. Along with the rapid development of information technology, China has gradually entered the "Internet +" environment, great importance has been given to "Internet +" industry development in our country, the "Internet + " health care is an important development direction. This form of development is popular and welcomed by the health care industry, the civilian battalion hospital actively use the environment facilitates people's registration, visits and other procedures. But there are some problems in private hospital financial management aspects, hinder the healthy and sustainable development of the hospital. This private hospital financial management should actively respond to climate change, some of the financial management innovation strategy is put forward.
\end{abstract}

\section{Introduction}

Internet and medical application is called the combination of the Internet, the traditional medical information collection, detection, diagnosis and treatment in the process of connected to the Internet through intelligent medical equipment, etc. Hence, relevant disease information within the hospital can break through the paper, and then realize the free flow, upload and share. Patients can also consult the doctor in time, thanks to the Internet, break through the geographical constraints, greatly facilitate the people's hospital. Itself should be analyzed under the new situation, civilian battalion hospital financial management problems, and then gives the corresponding innovation strategy.

\section{The operation mode and financial management requirements of the civilian battalion hospital under the "Internet+" environment}

The operation mode of the private hospitals under the "Internet + environment" .At present, the majority of mobile medical enterprise has yet to form a relatively clear profit model, is a waste of money. These medical institutions operating profit model is mainly: patients in Baidu or other familiarizing website free ask the doctor, the doctor on the online answer, the doctor through inquiring platform, for a price, which received praise from word of mouth is also a kind of intangible assets. The number of visits Internet platform to enjoy free services is still in the majority, payee less [1]. Internet medical is mainly composed of the patients, doctors, hospitals and pharmacy, etc, in which patients may pay for their medical consultation, in accordance with the reason they should pay for it, but the majority of Internet users in the patient's ideology, the Internet represents a free, so the possibility of paying very little. Other forms of payment mode is still not mature, is still in exploration. Internet medical operations and stratum another purpose is to attract as many industry personnel involved, make the patient and the doctor set up medical favor and trust of the Internet, and gradually cultivate the habit of online medical patients. Also can fully with the analysis of large data, understand user basic health conditions and requirements.

Financial management requirements of private hospitals under the "Internet +"environment. Most hospital's management most is that some older medical experts, the experts thought more traditional conservative, lack of enthusiasm for the new matters, also lack of money and effort of will, passive reception to information technology as well. Face + Internet, civilian battalion hospital should actively improve their financial management, and actively to the development of the Internet online, further development and input to the medical cloud data, implement positive promotion for mobile medical equipment, to invest in medical building, on the Internet should be 
increasing competition in medical areas [2]; Moderately to entities in the hospital medical resources open to Internet medical enterprise, integration of offline online resources in medical industry.

\section{The brief introduction of the civilian battalion hospital financial management}

Civilian battalion hospital financial management is mainly refers to all contained in the process of capital operation activity; its content is mainly in cost management, financial analysis and internal control management and so on. Its financial management goal is to use the least money and the cost to maximize capital and social benefits, is to achieve maximum economic benefits. Hospital financial management mainly includes the contents of these: raise money management, raise funds and use it daily in a hospital often and development, and channels to raise the medical income and shareholders' investment, etc.; Capital budget management, the hospital income and economic exchanges should be included into the financial budget, analysis of financial budget to guide the behavior of hospital operation and financial management; Revenue management, planning and management of hospital income and benefits; Cost management, the collection and distribution of medical costs, expenses and medical costs, control the cost and enhance the core competitiveness of their own; Asset management, the management of hospital fixed assets, current assets and intangible assets [3]. Financial analysis and reporting of key financial indicators to enhance analysis, timely write financial reports; Financial supervision and risk control, through the financial supervision and control risk, strengthen supervision on hospital economic activities, to ensure that funds reasonable distribution of science, to better the implementation of financial risk control. In order to show the work of financial department to the straightforward application process, now in the form of a chart showing the financial department budget management procedure, as shown in figure 1.

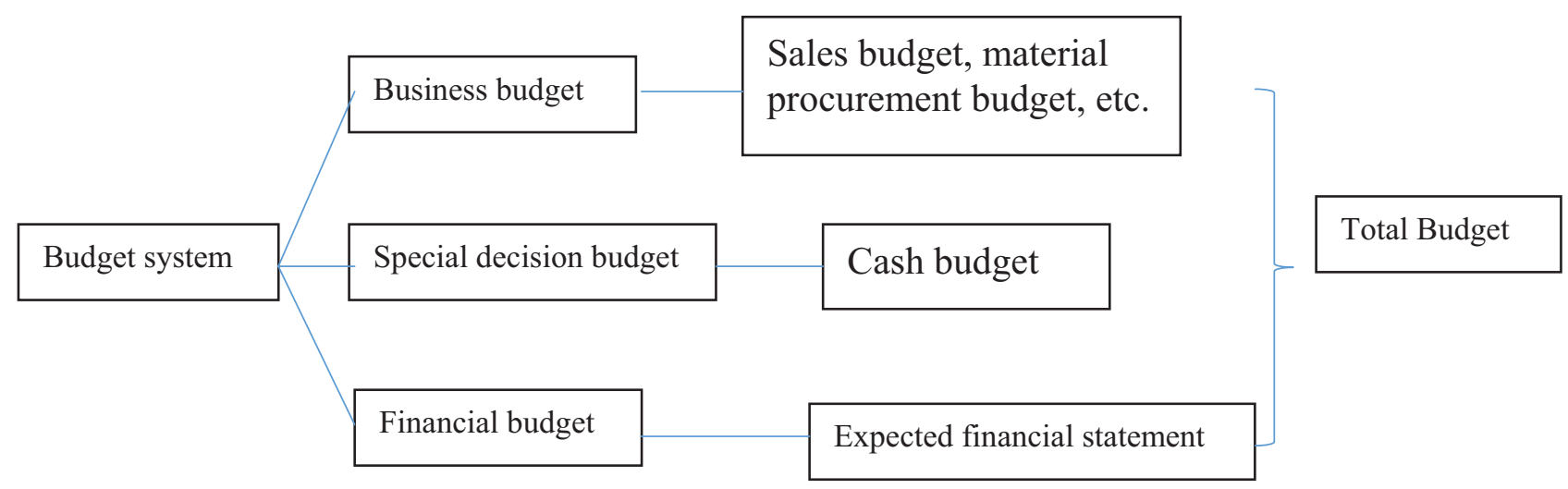

Figure 1. Budget System

\section{Analysis of the main problems in financial management of private hospitals}

Ignore the financial management, set up reasonable financial position .The civilian battalion hospital excessive emphasis on the doctor's selection and training, lack of attention to financial management behavior, which makes the hospital financial management, is chaotic behavior. Hospital financial position setting is not science; there is deviation on the financial management theoretical knowledge. Financial management personnel from hospital, no just implemented accounting, are not really achieving the goal of financial management. Financial personnel only served for the capital income and accounting records, in the aspect of cost control, direction control was not well done; its financial management level is low. Some civilian battalion hospital financial position setting is not strictly carried out in accordance with the accounting law, toll collector part-time cashier; asset managers do part-time archivists, and hired no accounting qualification of the personnel to be relevant.

The construction of the financial system is not perfect with poor execution .Sound financial management system is the necessary premise of hospital financial management, the main financial 
and accounting system of accounting, check the seal preservation and internal control system, etc. Some private hospitals and no financial system, and some even if it does, it is very imperfect, cause the hospital financial personnel's responsibility is not clear, the responsibility is not clear, and so on. System exists in the phenomenon of poor execution, system of some civilian battalion hospital will hang on the wall, not the actual execution, majority is to facilitate shall prevail.

The quality of financial personnel remains to improve .Now is the era of information technology rapid development, civilian battalion hospital financial management methods also should keep pace with The Times, keep up with the pace of the information age, the best use of electronic information technology for financial management innovation, but the reality is most of the financial management is still behind the traditional concept, there is no active learning new theoretical knowledge, cannot meet the requirement of hospital financial management, the low level of business [4]. After some financial personnel engaged in accounting work, ignore the business study, of the revised accounting standards, still use the old standard, poor work sense of responsibility, quality and work attitude.

Accounting information level and the quality is low. With the development of computer technology and the widespread use of accounting software, if the hospital use financial management software, will effectively improve the working efficiency of the financial staff, to promote the quality of financial work. But some private hospitals do not attach importance to accounting information, make some management accounting and financial management software is not popular, financial management personnel work efficiency is low, spent a lot of time. In addition, the accounting information quality is low, the quality of financial analysis, in turn, is poor, unable to hospital financial conditions play a role of good supervision and some financial information will not be able to fully submitted in a timely manner.

\section{Innovation strategy of civilian battalion hospital financial management under the environment of "Internet +"}

Increase the risk of civilian battalion hospital financial management consciousness. Civilian battalion hospital financial management department to deal with all the staff to implement the education of risk awareness and transmission, hospital staff only remained firmly in mind the risk consciousness, will reduce the risk in daily work, improve work efficiency, and further improve the quality of financial work in the hospital. The cultivation of risk for the hospital financial staff, on the one hand can improve the work quality, improve the ability to resist risks, financial sector and reduce the probability of financial problems. Hospital to risk awareness training on financial personnel, should from the actual situation with hospital financial situation, to develop a detailed training plan, be targeted to improve staff quality and skill [5]. In addition, the hospital should coordinate operational risk and the overall efficiency, to reduce the risk and to promote the progress of hospital development, handle the relationship between the opportunities and challenges, and realize its own development and growth.

Build a comprehensive budget management system and early warning mechanism. At present, although some private hospitals in China established the financial budget management system, but only to budget of income and expenses, do not belong to the category of comprehensive budget management. Comprehensive budget management is mainly of the real, control, execution and early warning of budget. The construction of a comprehensive budget management system, can lower the risk of financial management of private hospitals, and effective analysis, and to advance the potential risk. Private hospitals should gradually establish the cost of capital, income, expenses and suggests the budget, etc., effectively improve the financial budget management ability. With the aid of the budget management system and the financial risk early warning model, further enhance the financial management risk prevention and early warning of civilian battalion hospital, and to ensure timely find financial management risk and gives [6]. Hospitals in the building of the financial early-warning index system, we should, in accordance with the principle of systematic, scientific and 
comprehensive financial early warning indicators for scientific selection, and timely and effective control of risk.

Develop a perfect private hospital financial rules and regulations . Private hospitals should do a good job in financial rules and regulations construction to further strengthen the financial and accounting foundation work, actively on the basis of a new system of financial management and hospital accounting system, scientific and reasonable financial management positions, and financial personnel to set up and configuration, in view of the hospital budget management, cost control, expense reimbursement and set up a special system, makes the hospital those responsible had a clear, strictly according to the relevant responsible persons responsibility system investigated. Further build hospital performance evaluation system and incentive constraint mechanism, strengthen asset management, a more reasonable allocation of civilian battalion hospital resources. Hospital relevant financial personnel to cope with the financial system for regular or irregular assessment and examination, find out the weak links that exist in the system, in time to avoid system useless without work. Private hospitals in order to get a better development, the financial department will need to set some reasonable regulatory system and cost control regulations of the specification, analysis of financial indicators, reflected the real financial value to hospital, the hospital management sales and spending detailed results calculated [7]; Finance department should actively in regulatory department staff attitude and manner of work, formulate the corresponding measures of rewards and punishments to fully arouse the enthusiasm of the staff; Finance department shall also formulate rules for the various cost control, to control the cost of output, fine management costs, reduce cost, better for the welfare of the hospital.

Promote the informatization level of private hospitals . Our country has gradually entered the information age, information technology, more and more widely used in various industries, civilian battalion hospital should be combined with the era of social demand, analyze their advantages and disadvantages, for financial informatization construction in a planned way, to enhance the hospital hardware infrastructure construction, money for buying new financial management system, the traditional backward management software to optimize income, reduce the burden of work and financial workers. Financial staff should use advanced financial management software, science hospital financial management, improve capital operation rate, and improve the hospital financial management level and overall strength.

Create a good internal control environment . Private hospitals should learn advanced experience in financial management work, actively in hospital to create a good internal management environment, encourage financial management department to clear the importance of their work, actively advocate called for the hospital staff cooperate with finance department in management. Daily operations of the hospital departments should be detailed statistics and inherent assets, and the monthly accounts with financial personnel controls and regulations all cash flow should be assigned to the finance department is responsible for, strict internal supervision system in hospital, to prevent the department in charge of secretly.

\section{Summary}

Medical institutions are closely related to people's lives and health, its shoulders important mission and responsibility. Internal financial management efficiency of some private hospitals in our country is low, thus the hospital management efficiency is low. Financial management not value, even ignore the internal control, lack effective planning of capital and profit, making civilian battalion hospital face greater risks and challenges in the process of operation. If want to occupy a place in the fierce competition, private hospitals should be positive and keep up with the Times. Under the environment of Internet + , civilian battalion hospital of innovating financial management method strengthen the financial management of risk awareness, build a comprehensive budget management system and early warning mechanism, making perfect civilian battalion hospital financial rules and regulations, so as to promote the informatization level of civilian battalion hospital, etc. 


\section{Acknowledgements}

The general project, Hunan Provincial Department of education scientific research at the provincial level, "Internet plus under the background of the private hospital medical service model innovation research", No. 16C0193.

\section{References}

[1] Xu Dongsheng. "Internet + " environment private hospital financial management research [J]. Journal of economic management: abstract), 2016 (8): 00105-00105.

[2] Liu Yarong. Under "Internet +" in private hospitals to strengthen financial management research [J]. Chinese and foreign entrepreneurs, 2016 (16).

[3] Yang Guangyu. Small and medium-sized enterprise financial management mode under the network environment of innovation strategy [J]. Journal of global market, 2016 (4): 16-16.

[4] Mou Qunying. Analyses the pattern of small and medium-sized enterprise financial management innovation under the network environment [J], 2014 (16): 85-85.

[5] Chen Sijun. Introduction to financial management under the Internet environment [J], 2016 (26): 11-11.

[6] Wang Lihua. About the exploration of civilian battalion hospital financial management innovation [J]. Journal of management, 2013 (19).

[7] JiaoYundi, Liuying. Shallow of civilian battalion hospital financial management [J]. Modern economic information, 2011 (10) : 146-146. 